Res Pública Revista de Historia de las Ideas Políticas

ISSN: 1131-558X

\title{
L. F. Blengino, El pensamiento político de Michel Foucault, Madrid, Guillermo Escolar, 2018, 351 pp.
}

No siempre que algo genera una intensa e ingente polémica es un signo de la magnitud de su relevancia. En el caso de Michel Foucault, sin embargo, si algo parece evidente tras la publicación de los cursos del Collège de France en las últimas dos décadas, es que se trata de un autor central para pensar e investigar cuestiones relativas al fenómeno del neoliberalismo y al momento histórico que nos ha tocado vivir en términos políticos y sociales. Sea para apoyarse en él o para criticarlo, Foucault se ha vuelto una figura casi ineludible de los debates que configuran nuestro presente.

El libro de Luis Félix Blengino asume el reto de utilizar a Foucault para abordar dos problemas que atraviesan la reflexión y la praxis políticas contemporáneas con una intensidad quizá acorde al grado de lo que está en juego para quienes la transformación social y la emancipación siguen siendo un horizonte no sólo deseable, sino necesario. Por un lado, la configuración del neoliberalismo como una situación de dominación en que las relaciones de poder adquieren "un grado tal de inmovilidad y fijación que amenaza con convertir en una quimera cualquier posibilidad de subversión del orden de lo dado" (p. 244). Por el otro, un debate recurrente que, a pesar de tener a sus espaldas un largo recorrido a través de distintas fases, a veces parece enquistarse en presupuestos y dicotomías tan problemáticas como inoperantes: la relación entre las luchas en torno a la subjetividad y las luchas en torno a la dominación y la explotación. A partir de estas dos problemáticas, el libro se organiza en dos partes que se articulan alrededor de un capítulo (pp. 217-248) dedicado a plantear un diagnóstico de la situación presente de la dominación a partir de la cartografía foucaultiana de la gubernamentalidad neoliberal como gubernamentalidad "omnímoda e ilimitada" (p. 221).

La primera parte (pp. 77-215) constituye una concienzuda reconstrucción de los dos cursos impartidos por Foucault en los años 1977-1978 y 1978-1979, bajo los títulos respectivos de Seguridad, territorio, población y Nacimiento de la biopolitica, con el objetivo de mostrar el hilo conductor que, según Blengino, constituye uno de los núcleos fundamentales de la ontología de nuestro presente: "la gubernamentalización del Estado" (p. 45). La segunda parte (pp. 249-311) se mantiene en esos mismos años, pero aborda una parte distinta del corpus textual foucaultiano que no ha sido menos polémica: los escritos sobre la revolución iraní. Tras la reconstrucción llevada a cabo en los capítulos anteriores de esa tendencia a la gubernamentalización del Estado,
Blengino amplía el marco para mostrar en los escritos iraníes "el momento fundamental de la reflexión foucaultiana acerca de la resistencia como movimiento de liberación" (p. 285) y establecer un punto de conexión entre lo que había sido el trabajo previo de esos cursos de 1977-1978 y 1978-1979 y las investigaciones posteriores en torno al cuidado de sí o la parrhesía.

Si una de las cosas que hacen de la publicación de este libro en la colección "Euroamericana" de Guillermo Escolar un acierto editorial es su voluntad de insertarse en la discusión de asuntos políticos de máxima actualidad, la otra es que constituye una importante contribución a los debates académicos en torno a la interpretación del trabajo histórico-filosófico de Foucault y una solvente toma de partido en algunas de las polémicas surgidas en el seno de los estudios foucaultianos. Concretamente, las referidas al paso de la concepción bélica del poder ("hipótesis Nietzsche") al problema del gobierno y a la caracterización y justificación tanto de la resistencia como de su sujeto.

La hipótesis de partida que adopta Blengino para insertarse en estas discusiones es que "la progresiva gubernamentalización del Estado, es decir, la constitución del triángulo seguridad-economía política-población, indagada a la luz de la relación disimétrica establecida entre Europa y el resto del mundo, es la clave para comprender la racionalidad de gobierno liberal en términos de dominación, explotación y sujeción-subjetivación" (p. 24). Esta perspectiva geopolítica que destaca José Luis Villacañas en el prólogo es una de las aportaciones que se separa de la lógica que ha determinado los centros de interés y atención dedicados al pensamiento político de Foucault desde la publicación de sus cursos. Si habitualmente se destaca la novedosa matriz de inteligibilidad a la hora de pensar el poder, problematizar la distinción conceptual Estado-sociedad o analizar las técnicas de gobierno y los regímenes biopolíticos, la mirada sobre el plano internacional permite a Blengino, entre otras cosas, avanzar una tesis importante en lo que se refiere a la discusión acerca de la concepción foucaultiana del neoliberalismo. Como señala el propio autor, el argumento principal del libro busca mostrar "el modo en que el neoliberalismo se constituye, de acuerdo con la cartografía histórica foucaultiana, en un arte de gobernar que tiende -aun fundándose en la producción y consumo de libertades- a constituirse en una situación de dominación sin resto aparente, i. e., en un estado de dominación, puesto que implica un modo de ejercicio ilimitado del poder en todas las direcciones que adopta 
su orientación: una expansión ilimitada hacia el exterior del Estado y de la región geopolítica dominante y una gubernamentalidad máxima hacia el interior a través de una tecnología ambiental y de subjetivación capaz de abarcar la totalidad de las conductas humanas como si fueran conductas de empresarios de sí” (p. 25).

Defender esta tesis implica un determinado posicionamiento interpretativo que sea capaz de sostener una lectura en cierto modo a la contra de la recepción dominante de los escritos de Foucault. Por eso el primer capítulo del libro (pp. 29-48) está dedicado a lograr un equilibrio metodológico allí donde otros planteamientos demasiado unilaterales han condicionado una determinada visión, por un lado, respecto al supuesto abandono de la comprensión bélica del poder en favor de una consideración del gobierno en cuanto conducción de conductas y, por el otro, del tipo de ruptura que se produce entre los cursos de 1977 y 1978 y la orientación de su investigación en los cursos anteriores.

Respecto a lo primero, Blengino aprovecha la perspectiva privilegiada que le aporta la adopción de la escala geopolítica para pensar en la concepción foucaultiana del poder no solo desde el problema de la constitución política interna de las sociedades modernas, sino en relación al vínculo de Europa con el resto del mundo. Esto es lo que le permite señalar cómo el consenso más o menos general acerca del abandono del modelo de la guerra como matriz de inteligibilidad del poder se asemeja demasiado a la autoconcepción de sí de las sociedades modernas que Foucault trató de deshacer con tanto denuedo. La idea de que Foucault habría reemplazado la matriz bélica por la categoría de gubernamentalidad converge con el presupuesto de la autoconcepción liberal en el momento en que reproduce el esquema de un progresivo abandono de formas arcaicas de conflicto (la guerra) por formas de interacción más civilizadas (comercio, gobierno económico, etc.). Más que un reemplazo, Blengino apuesta por la idea de una ampliación de la hipótesis Nietzsche con el objetivo de abordar el problema de la libertad. Solo así es posible no volver a dejar de lado cuestiones como la conquista o la colonización. La inteligente propuesta del libro busca integrar ambas dimensiones o modelos de análisis (la guerra y el gobierno) para pensar cómo habría que entender el conflicto y la dominación bajo un "contexto teórico dominado por la categoría de gubernamentalidad" (p. 39).

En lo que se refiere al tipo de ruptura que se produce a partir del curso de 1976, Blengino critica la unilateralidad de interpretaciones como la de Stephen Collier. La apuesta por un abandono definitivo de todo tipo de diagnósticos globales o lógicas totalizantes en favor del análisis de formas contingentes de correlación de elementos no tomaría en suficiente consideración el concepto de "sistemas de dominantes". El punto fundamental señalado por Blengino es que sin la dimensión histórica diacrónica que subyace a dicho concepto no se puede captar el tipo de continuidad y variación que cualifica las distintas correlaciones bajo la categoría de la gubernamentalidad. No solo no se podría justificar la prioridad otorgada por Foucault al problema del gobierno y al proyecto de la historia de la gubernamentalidad. Tampoco estaríamos en condiciones de utilizar provechosamente los resultados de la genealogía foucaultiana para dar cuenta de la gubernamentalización del Estado como un proceso determinante en las sociedades contemporáneas.

Desde este punto de vista, este libro es una importante contribución a la hora de asentar una perspectiva que integre los dos polos de ambas cuestiones: por un lado, una matriz de análisis del poder que acoja tanto el modelo bélico como el modelo gubernamental y se haga cargo de la doble dimensión del conflicto y la libertad; por el otro, una perspectiva metodológica al mismo tiempo topológica y epocal que no disponga los diagramas de poder de manera rígida en una clave dialéctica secuencial, pero que tampoco excluya ni las variaciones diacrónicas ni la consideración de la prioridad que pueda adoptar un elemento en una determinada combinación de las disposiciones correlacionales.

El hecho de que Blengino busque sacar a la luz mediante la perspectiva diacrónica el hilo conductor que subyace a la historia de la gubernamentalidad no es arbitrario. Mostrar la progresiva constitución estratégica de una "forma de poder simultáneamente totalizante e individualizadora" (p. 81) no solo busca clarificar aspectos importantes de la cartografía foucaultiana del poder moderno. También es un posicionamiento respecto a las consecuencias de dicha cartografía. Un intento de hacerla operativa, sin evitar tomar partido en las polémicas interpretativas, impulsado por la preocupación de ofrecer un diagnóstico acertado que guíe la resistencia a una dominación neoliberal cuya actualidad hunde sus raíces en la integración del poder pastoral en las formas de racionalidad del poder del Estado moderno.

Como se ha dicho antes, la perspectiva que aporta el plano internacional, específicamente "la emergencia de Europa como sujeto geopolítico moderno y como región vinculada de una manera singular con el resto del mundo" (p. 92), constituye una de las contribuciones más propias del libro. El nacimiento del liberalismo y su posterior reconfiguración neoliberal aparecen marcados por la continuidad de la dominación económica, la utilización comercial y la colonización, desmintiendo así la idea de que Foucault habría elaborado una cartografía del poder sin dominación. Mediante la combinación de las matrices de inteligibilidad epocal-topológica y bélico-gubernamental, y atendiendo al "quiasmo relativo a los planos interno e internacional" (p. 114), Blengino muestra convincentemente que la inversión del signo de los objetivos de la intervención liberal respecto a la razón de Estado es doble y que no se puede separar la nueva limitación intraestatal del nuevo imperialismo y de la mundialización del mercado como forma de desplazar la suma cero del juego económico lejos de los equilibrios intraeuropeos.

Aunque las consideraciones de Foucault acerca de la singular dimensión internacional del neoliberalismo son menos extensas, las menciones referidas a la Comisión Trilateral, a la legitimación política del nuevo Estado alemán por la libertad de mercado y a la aplicación de la teoría del capital humano al análisis del desarrollo desigual permiten atender a la fase neoliberal de la gubernamentalización del Estado sin perder de vista el 
plano geopolítico. En este sentido, resulta especialmente interesante el señalamiento de cómo la teoría del capital humano ha permitido recuperar un esquema de desarrollo histórico basado en la experiencia de modernización europea que, al mismo tiempo que omite los bloqueos estructurales de las políticas de desarrollo, reformula la subordinación del Tercer Mundo bajo la idea del subdesarrollo (pp. 151-153).

En todo caso, la atención a la dimensión internacional no priva a la reconstrucción de la cartografía foucaultiana que lleva a cabo Blengino de interesantes aportaciones también en lo que se refiere al análisis de las transformaciones sociales implicadas por la gubernamentalidad neoliberal. Como se mencionaba al principio, uno de los principales objetivos del libro es mostrar la tendencia a establecer una forma de gobierno omnímoda e ilimitada. Blengino destaca con mucho acierto que la intervención del neoliberalismo norteamericano busca superar la ambigüedad ordoliberal, fruto de la necesidad de perseguir "por un lado, la producción de la sociedad de empresa y de los sujetos competidores y, por el otro, la compensación de los efectos disolventes de la competencia a través de la producción activa de lazos comunitarios" (p. 176), mediante la teoría del capital humano y la ampliación ilimitada del concepto de homo economicus. Lo interesante de la interpretación es haber detectado la importancia de la teoría del capital humano como un marco de inteligibilidad de las conductas que permite incluirlas en el cálculo y la programación económicas independientemente de su racionalidad o irracionalidad desde este punto de vista. Esta limitación de los objetivos de las tecnologías de subjetivación, que no busca ni producir un nuevo sujeto competitivo ni defender una antropología naturalista, es la radicalización que confiere al enfoque de la tecnología ambiental un alcance prácticamente ilimitado que amenaza con condenar al fracaso toda forma de resistencia.

Este es el tema que articula la segunda parte del libro. Merece la pena destacar el valioso análisis de la célebre entrevista concedida por Foucault el 20 de enero de 1984 (pp. 234-248), publicada con el título "La ética del cuidado de uno mismo como práctica de libertad". Blengino ofrece una interpretación de los pares conceptuales "estado de dominación-liberación" y "relaciones de poder-prácticas de libertad" extremadamente útil para esclarecer algunos de los problemas en que se juega el sentido de la categoría de resistencia, posicionándose en los debates acerca del último Foucault tratando de calibrar hasta qué punto la introducción de ciertas distinciones en los mencionados pares conceptuales permiten considerar o no la gubernamentalidad neoliberal como una situación de la que sea necesario "liberarse" o si más bien estaríamos ante una práctica de libertad que permite únicamente prácticas de resistencia en relación con uno mismo. A partir de la admisión por parte de Foucault de la posibilidad de que se den en un estado de dominación prácticas de resistencia que, al no lograr revertir las relaciones de poder, devienen una suerte de gesto subversivo inocuo, Blegino rescata la introducción de una diferencia tanto entre los conceptos de prácticas de libertad y prácticas de resistencia como en las formas mismas de resistencia. Su tesis fundamental es que cabe una cierta matización del sentido absoluto e ideal de los conceptos de dominación y liberación de manera que se entienda la primera no solo como concepto límite que delimita por un extremo el campo de las relaciones de poder ("estado de dominación"), sino también como relación de poder relativamente fija e inmóvil ("situación de dominación") en la que se puede distinguir entre resistencias que constituyen meras "astucias" y prácticas de resistencia capaces de desbloquear dichas relaciones de poder y alterar la estructura de la situación de dominación ("prácticas de liberación").

El nuevo par conceptual alcanzado por Blengino -"situaciones de dominación" y "prácticas de liberación"- abre las puertas al análisis de los escritos sobre la revolución iraní para dilucidar en qué sentido se puede hablar de la importancia otorgada por Foucault a los modos de subjetivación como punto de partida imprescindible en las luchas políticas y económicas contemporáneas. Lo reseñable del abordaje de unos textos tan polémicos por parte de Blengino tiene que ver, primero, con situarlos en el contexto de lo que supusieron para Foucault las experiencias del viaje y el periodismo. Ello implica no tratar los escritos iraníes como desviaciones puntuales de su práctica histórico-filosófica, sino como un hito clave en su propio descentramiento y desidentificación como sujeto. Es esta contextualización lo que permite establecer los artículos publicados en Corriere della Sera, Le Nouvel Observateur y Le Monde como clave interpretativa de la problematización foucaultiana de la idea de revolución.

Lo interesante del punto de partida de Blengino es que asume la caracterización que hizo Badiou de los escritos iraníes como un "error aparente" (p. 278). El rechazo de la idea de que la toma de posición de Foucault respecto a la revolución iraní fue una equivocación o un absurdo busca restaurar tales textos como partes de pleno derecho del corpus foucaultiano. Pero Blengino se aleja también de aquellas interpretaciones como la de Judith Revel que, aun asumiendo la plena complejidad de estos textos y su lugar dentro del proyecto filosófico de Foucault, tratan de salvar a éste del contenido explícito de estos textos sosteniendo que su interés por la revolución en Irán tenía más que ver con su condición de "acontecimiento" (p. 299) que con sus características. Para Blegino, la revolución iraní está "vinculada con los cuatro umbrales de modernidad expuestos en el primer capítulo, pero también con la ficción histórica que se delineó a partir de la tendencia de gubernamentalización del Estado que ellos inauguran" (p. 284). Es decir, se trataría de un acontecimiento en que Foucault capta elementos decisivos que están en relación directa con el desarrollo de su pensamiento político no porque emerja al margen de cualquier lógica, sino precisamente porque constituye un proceso que forma parte de la cartografía y el diagnóstico epocal de una situación geopolítica e histórica concreta: "el arte neoliberal de gobernar en la racionalidad económica de los gobernados" (p. 300).

Se puede decir que El pensamiento político de Foucault logra conjugar una pormenorizada exposición de la historia de la gubernamentalidad, atendiendo a los deba- 
tes hermenéuticos y justificando un posicionamiento en temas tan polémicos como el modelo de análisis del poder en el plano metodológico o la relación entre dominación, resistencia y liberación en plano teórico, con una propuesta teórica actualizada en relación a las discusiones políticas contemporáneas a partir de la cartografía foucaultiana del poder. Además, la específica atención a la dimensión geopolítica le otorga una perspectiva privilegiada a la hora de abordar el problema de la modernización desde las herramientas analíticas foucaultianas. Todo ello convierte este trabajo en una importante contribución a los estudios de la obra de Foucault y una lúcida intervención en la coyuntura política que marca nuestro presente.

Jorge del Arco Ortiz 